\title{
Rivages totémiques
}

Totemic shorelines

\section{Marie Mauzé}

\section{(2) OpenEdition}

\section{Journals}

Édition électronique

URL : http://journals.openedition.org/span/1583

DOI : $10.4000 /$ span. 1583

ISSN : 2268-1558

\section{Éditeur}

École pratique des hautes études. Sciences humaines

\section{Édition imprimée}

Date de publication : 1 décembre 1998

Pagination : 127-168

ISSN : 0294-7080

\section{Référence électronique}

Marie Mauzé, «Rivages totémiques », Systèmes de pensée en Afrique noire [En ligne], 15 | 1998, mis en ligne le 02 juin 2014, consulté le 30 avril 2019. URL : http://journals.openedition.org/span/1583 ; DOI 10.4000/span.1583

(c) École pratique des hautes études 


\title{
RIVAGES TOTÉMIQUES
}

\author{
par
}

\section{Marie Mauzé}

Nous nous proposons d'examiner comment la problématique du totémisme a été introduite dans l'étude des cultures de la côte NordOuest de l'Amérique du Nord. L'expression " côte Nord-Ouest " désigne à la fois une aire géographique et une aire culturelle. L'aire géographique s'étend sur plus de deux mille kilomètres de littoral et d'immédiat arrièrepays, de la baie de Yakutat en Alaska, au nord, jusqu'au fleuve Columbia, qui sépare les Etats américains de Washington et d'Oregon, au sud; en font partie la totalité de la façade océanique de la province canadienne de la Colombie britannique, avec l'archipel des îles de la Reine Charlotte, les îles du Prince-de-Galles et l'île de Vancouver. L'aire culturelle correspondante réunit les territoires des sociétés nord-amérindiennes traditionnellement distinguées par les anthropologues que sont, du nord au sud : les Tlingit, les Haida, les Tsimshian, les Gitksan et les Nishga, les Kwakiutl septentrionaux (Haisla, Heiltsuq et Owikeno), les Bella Coola, les Kwakiutl méridionaux, les Nootka, les Salish de la côte et de l'intérieur'.

${ }^{1}$ J'utilise ici les ethnonymes que l'on connaît dans la littérature anthropologique et non ceux localement en usage aujourd'hui.

\section{Totémismes}

Systèmes de pensée en Afrique noire, 15, 1998 
Le débat sur l'existence d'un totémisme a principalement porté sur l'ethnographie des Tlingit, des Haida et des Tsimshian; il n'a concerné que secondairement les $\mathrm{K}$ wakiutl, en dépit de l'importance de la littérature qui leur a été consacrée : nous pensons bien entendu à l'œuvre de Franz Boas. Dans ce qui suit, après avoir présenté les grands traits de l'organisation sociale des trois sociétés septentrionales, nous rappellerons rapidement dans une première partie ce qu'a été le cheminement des recherches sur le totémisme pour la région considérée, puis nous en viendrons à la problématique fondamentale du " blason " (crest), qui fait signe de l'existence d'une institution que l'on peut juger être plus ou moins lointainement de type totémique mais dont l'analyse peut être faite indépendamment de tout a priori interprétatif renvoyant au modèle du totémisme qui a prévalu dans la littérature anthropologique classique.

L'ethnographie de la région est vieille de plus d'un siècle et a mobilisé les efforts d'un grand nombre d'anthropologues, dont quelquesuns des plus grands (Boas, Swanton, Murdock, Sapir, Garfield, de Laguna, etc.), qui ont travaillé différemment, à des époques différentes et bien entendu sur des terrains différents, de telle manière qu'on ne peut leur tenir rigueur d'utiliser pour chaque contexte d'enquête et d'analyse des vocabulaires descriptifs partiellement distincts. Nous nous efforcerons d'introduire dans ce vocabulaire un certain nombre de simplifications qu'on voudra bien ne pas considérer comme réductrices : elles ne visent qu'à une clarification d'ensemble que les spécialistes pourront critiquer mais dont nous espérons que les lecteurs peu familiers des sociétés évoquées tireront bénéfice en matière de lisibilité de faits dont le détail est particulièrement complexe.

A la différence des autres sociétés de la côte Nord-Ouest, les Tlingit, les Haida et les Tsimshian sont des sociétés matrilinéaires. L'identification des unités de référence implique la distinction de trois niveaux de réalité sociale allant du plus général au plus spécifique, le premier et le troisième niveaux étant représentés dans chacune des trois sociétés, tandis que le niveau intermédiaire n'est attesté que chez les Tlingit. Pour aller à l'essentiel, le premier niveau est celui qui correspond à une division de la société en deux moitiés exogames ou en quatre " quarts ", tandis que le troisième est, de manière contingente, celui des plus petites unités sociales de base. 
Les Tlingit et les Haida se répartissent en deux moitiés (appelées aussi " phratries" ou " clans " dans la littérature). Les moitiés tlingit sont celles des Corbeaux et des Loups ${ }^{2}$, les moitiés haida celles des Aigles et des Corbeaux. Il est entendu qu'un individu tlingit, homme ou femme, est soit un Corbeau, soit un Loup, qu'un individu haida est soit un Corbeau, soit un Aigle; dans les deux cas, l'appartenance à une moitié se transmet en ligne matrilinéaire. Les moitiés sont exogames, chacune d'elles jouant le rôle de moitié opposée à l'occasion d'un certain nombre de prestations de services, notamment dans les contextes cérémoniels, plus particulièrement lors des funérailles. Si, d'une manière générale, les moitiés sont désignées par des noms d'espèces naturelles, ce qui leur a valu l'appellation de "groupes totémiques ", le système d'appellation des lignages de la côte Nord-Ouest ne fait pas obligatoirement appel à des noms d'animaux : ainsi clans et lignages portent-ils souvent des noms de lieux, à l'instar, par exemple, des Ganaxadi, clan de la moitié Corbeau des Tlingit, qui tiennent leur nom d'une île (Swanton, 1908).

A ce premier niveau, les Tsimshian diffèrent des Tlingit et des Haida en ce qu'ils connaissent une division non pas bipartite mais quadripartite, les quatre fractions étant généralement appelées " clans ". Les noms des quatre clans tsimshian reproduisent ceux des deux moitiés tlingit et haida (Corbeau-Loup, Aigle-Corbeau) auxquels s'ajoute celui de l'Epaulard ${ }^{3}$. Les clans tsimshian sont exogames. La distribution dans l'espace de leurs membres est telle qu'on ne trouve jamais plus de deux clans représentés dans une même communauté locale. Tout se passe alors comme si la pratique sociale renvoyait à un système de moitiés. A titre de comparaison, indiquons que chez les $\mathrm{K}$ wakiutl, le premier niveau, étranger au système des moitiés, est celui des groupes locaux ou " tribus".

${ }^{2}$ Chez les Tlingit du Nord, les Loups sont remplacés par les Aigles.

${ }^{3}$ Les clans des Tsimshian sont dénommés en fonction de leur blason principal : Loup (Laxgibu : litt. "sur le Loup "), Aigle (Laxsgiik, litt. "sur l'Aigle "), Corbeau (Ganhada, terme qui n'a aucune signification en langue tsimshian, mais qui pourrait avoir une origine tlingit ou haida), Epaulard-Grizzli (Gispwudwada, ce terme ayant à voir avec un nom de lieu : de Laguna, 1975, Garfield, 1951). Chez les Gitksan, on trouve les clans suivants : Epilobe (Fireweed) qui est l'équivalent de l'Epaulard des Tsimshian, Aigle, Corbeau et Loup. Chez les Nishga, seulement trois clans sont représentés. 
Ce que nous appelons le deuxième niveau n'est repérable dans l'ensemble septentrional que chez les Tlingit, où il rend compte de l'existence de " clans" matrilinéaires, qui, contrairement aux clans tsimshian, ne sont pas des produits de dérivation ou de déconstruction du système des moitiés, mais correspondent aux unités de base de la société, très précisément en ce sens qu'ils ne sont pas nécessairement subdivisés en unités plus petites, référées à notre troisième niveau.

Alors qu'au premier niveau ont été envisagés ensemble le cas des Tlingit et celui des Haida, en raison de la similitude de leur organisation en moitiés, l'examen des faits relatifs au troisième et dernier niveau conduit à opposer les Haida et les Timshian aux Tlingit. En effet, compte tenu que les clans tsimshian sont nés de moitiés, nous passons directement des moitiés haida et des clans tsimshian aux unités sociales de base des sociétés correspondantes, qui sont des matrilignages exogames (corporate matrilineages), auxquels on réserve simplement le nom de " lignages " pour les Haida, tandis qu'en référence au terme indigène, on parle de "maisons" pour les Tsimshian ; on trouverait une situation formellement comparable chez les $\mathrm{K}$ wakiutl, avec les deux niveaux des tribus et des maisons, ces dernières étant habituellement désignées par leur nom kwak'wala de numaym.

Si les Tlingit se singularisent par rapport aux Haida et aux Tsimshian, ce n'est pas principalement parce que nous $y$ distinguons deux niveaux "en dessous" du niveau des moitiés mais parce que l'existence du niveau 2 n'implique pas l'existence subordonnée d'unités de niveau 3, lignages ou maisons. On notera que dans le contexte d'ensemble considéré, l'emploi du mot " clan " relève de deux logiques distinctes, l'une liée à la génération des moitiés, l'autre à celle des unités sociales de base ; dans le premier cas, le clan n'est pas un groupe au sens sociologique du terme, dans le second cas, il est le groupe par excellence.

L'unité de base des groupes septentrionaux de la côte Nord-Ouest, qu'elle soit le clan chez les 'Tlingit, le lignage chez les Haida ou la maison chez les Tsimshian, est à la fois un groupe fondé sur la parenté et une personne morale. C'est une unité résidentielle - elle correspond à une ou plusieurs maisons au sens matériel du terme -, économique et politique. Tout clan, lignage ou maison a un patrimoine matériel et symbolique ou spirituel. Outre un territoire et des droits traditionnels d'usage et 
d'exploitation de ce territoire, ces unités possèdent aussi des noms attachés à des positions hiérarchiques, que seuls certains individus, en vertu de leur rang de naissance, peuvent occuper, ainsi que des blasons, des mythes, des chants, des danses, etc.

L'exogamie de moitié matrilinéaire ne se pratique pas seulement dans le cadre de la société identifiée par l'ethnonymie la plus englobante. On peut en effet parler d'un système régional - à l'échelle de l'aire culturelle - de moitiés exogames dont l'identification est fondée sur la détention de blasons. Le partage d'un même blason a valeur d'apparentement symbolique entre les partenaires concernés ; cet apparentement, que l'on peut à la limite considérer comme relevant d'une parenté pan-classificatoire, rend compte non pas d'une ascendance commune mais d'un savoir partagé renvoyant à une histoire plus ou moins commune (Boelscher, 1988 : 146). Ainsi les blasons principaux de la moitié haida Corbeau coïncident-ils avec ceux des clans tsimshian Epaulard et Loup, ainsi qu'avec ceux de la moitié tlingit Loup, alors que les emblèmes de la moitié haida Aigle occupent une position comparable à ceux des Corbeaux et des Aigles tsimshian et des Corbeaux tlingit (Swanton, 1905a : 65-66; Boas, 1916b). Il est interdit de se marier dans une moitié " amie " : un Corbeau tlingit ne peut épouser un Aigle haida (cf. aussi Dunn, 1984 ; Garfield, 1939 ; Seguin, 1984).

\section{Les débats autour du totémisme de la côte Nord-Ouest}

Le totémisme présente cette étonnante particularité qu'on en a théorisé la notion avant d'avoir véritablement exploité les matériaux ethnographiques susceptibles d'en induire l'existence. Par la suite, la succession des interprétations n'a pas toujours rendu compte des enrichissements de l'ethnographie. En même temps, toute ethnographie nouvelle ayant à voir avec le fait totémique réel ou supposé a été nécessairement " prise " dans un discours de caractère interprétatif. On peut aisément imaginer que cette imbrication de la production des faits et de l'interprétation n'a pas été pour rien dans le succès de l'hypothèse 
totémique ${ }^{4}$. Aujourd'hui, il paraît aussi illusoire de penser pouvoir livrer des matériaux " frais " allant dans le sens de cette hypothèse, qu'il s'agisse de la côte Nord-Ouest ou d'une autre région du monde, que d'en proposer une formulation unificatrice sur laquelle pourrait se faire l'accord des anthropologues.

La question du totémisme a occupé le devant de la scène anthropologique, tant en Europe qu'aux Etats-Unis, pendant une bonne quarantaine d'années, en gros de 1880 à 1920. L'apparition des premières formulations de la théorie du totémisme n'est pas séparable d'un contexte intellectuel de la fin du $\mathrm{XIX}^{\mathrm{e}}$ siècle fortement influencé par l'évolutionnisme. Le totémisme est alors considéré comme une institution représentative de la société primitive ; il rend compte d'une étape nécessaire de la formation de toute pensée religieuse (Voget, 1975 : 241 ; Wagner, 1987 : 573). La côte Nord-Ouest n'échappe pas à ce type de mise en perspective, même si cette région d'Amérique du Nord ne figure pas au nombre des aires culturelles les plus sollicitées à cet égard. On considère en effet que le totémisme de la côte Nord-Ouest est sur son déclin, comme l'indique le fait que le " totem " ne subsiste plus que sous la forme du " blason ", la relation du groupe à l'animal-totem ne s'établissant plus qu'à travers la référence au monde mythique (Durkheim, 1912 : 158 ; Goldenweiser, 1910).

Nous ne reprendrons pas dans le détail l'historique du * totémisme " depuis l'apparition du terme " totem " à la fin du XVII" siècle ( $c f$. par exemple, Haekel, 1983 ; Lévi-Strauss, 1962a \& b ; Testart, 1981 ; Wagner, 1987 ; Wintrop, 1991). On rappellera en revanche avec Paul Jorion (1985 : 861) que le totémisme, successivement ou simultanément, a été envisagé comme " une forme élémentaire de religion, ou un type d'organisation sociale, une forme de classification ou une cosmologie ". On notera encore qu'à un des tournants majeurs de la réflexion sur le totémisme sont associés les noms de Boas (1910b, 1916a \& b) et de

4 Rappelons la phrase de Marcel Mauss (1947 : 175) : " Après l'enquête de Frazer, le nombre de sociétés relevant de l'ethnographie qui ne connaissent pas le totémisme se réduit tous les jours". 
Goldenweiser (1910, 1918), ce qui nous fait retrouver la côte NordOuest $^{5}$.

Les deux auteurs cités considèrent que sous l'étiquette " totémisme " sont rassemblés des manifestations d'une très grande hétérogénéité. Goldenweiser remet en cause le caractère unitaire du totémisme en soulignant qu'il est erroné d'associer des faits tels que l'exogamie, les modalités de comportement par rapport à l'animal totémique, la conception d'une descendance de cet animal, qui sont indépendants les uns des autres. Boas (1916a : 321) souligne que * le totémisme est une unité artificielle et non naturelle ", propos que reprendra à son compte Goldenweiser (1918) ; il objecte qu'on puisse " déduire des lois psychologiques universelles" de " phénomènes anthropologiques qui sont en apparence semblables [mais] d'un point de vue psychologique tout à fait distincts" car les phénomènes totémiques ont chacun leur spécificité psychologique et historique (Boas 1916a : 323-324). Boas considère que " (...) sous l'influence d'une idée dominante des formes analogues peuvent se développer qui ont des origines différentes" (1916a : 321). Il trouve une illustration de son propos sur la côte Nord-Ouest où, rappelle-t-il, le substrat totémique tel qu'il se manifeste dans les tribus du nord de la Colombie britannique et dans celles du sud de l'Alaska n'est pas le même. L'uniformisation du totémisme telle qu'elle apparaît dans la région s'est accomplie par des emprunts d'idées et d'institutions entre groupes voisins.

A l'instar de Boas, Goldenweiser $(1910: 270)$ insiste sur le fait que * la spécificité du totémisme ne peut pas être découverte dans la somme des éléments tenus pour [en être] caractéristiques au sein d'une société donnée, mais dans la relation que ces éléments entretiennent les uns avec les autres (...). Il est évident, ajoute-t-il, que ce processus est une association [de ces éléments] et ne relève pas d'une simple juxtaposition ". L'observation est d'une importance capitale, car elle marque une tentative de séparation entre le contenu et la forme du totémisme. Goldenweiser remarque encore : "Le totémisme doit exprimer une relation entre des groupes sociaux et des symboles" (1910:275). Il

5 On peut se reporter à l'article de Shapiro (1991) pour une évaluation comparative et critique de l'apport de Boas et de Goldenweiser à la théorie du totémisme. 
explicite ainsi son propos: " Le contenu des traits [totémiques] de chaque clan est différent, mais la forme qu'ils revêtent est strictement identique dans toutes les unités du groupe, que l'on peut décrire comme des unités totémiques équivalentes" (1912a : 384). Même si les groupes orit des privilèges distincts, ce qui compte, c'est qu'ils exercent des fonctions non pas distinctes, mais équivalentes. Boas ne dit pas autre chose quand il note que les marques distinctives qui caractérisent les groupes exogames sont de même nature, tout en ayant un contenu différent :

" Il n'est pas possible que des traits distinctifs appartiennent à des domaines de pensée différents; qu’un groupe soit défini par un nom, un autre par un rituel, un troisième par des emblèmes ou des blasons (...). Nous devons conclure (...) que l'homologie des caractères distinctifs des divisions sociales d'une tribu prouve que leur usage a son origine dans une tendance à la classification "(Boas, 1916a: 326).

Ainsi que l'indique Claude Lévi-Strauss (1962b:18), on doit à Boas - et à Goldenweiser -, " le passage d'une définition concrète du totémisme à une définition formelle ". Le totémisme peut-il relever d'une définition univoque ? Gladys Reichard avait déjà répondu à cette question en son temps, en résumant la position des anthropologues d'obédience boasienne dans un article de l'ouvrage collectif General Anthropology, publié sous la direction de Boas. Reichard (1938 : 430) note avec justesse que le totémisme, tel qu'il a été décrit et défini, “ (...) se manifeste sous des formes si diverses (...) qu'il est absolument impossible de faire entrer celles-ci dans une seule catégorie ». A l'époque où ce texte est écrit, les anthropologues américains se sont déjà détournés du totémisme, dont l'étude occupera une place désormais marginale dans leurs travaux. Lévi-Strauss (1962a \& b) reprendra le dossier quelque trente années plus tard en convoquant les principaux théoriciens du totémisme. Il concluera que le totémisme n'est pas un phénomène ethnographique propre à certaines sociétés, mais une manifestation particulière d'une disposition intellectuelle universelle. La question centrale est la suivante : comment les hommes perçoivent-ils, ordonnent-ils et structurent-ils les ressemblances et les différences dans la société et dans son environnement naturel, et comment établissent-ils des relations entre l'ordre de la nature et celui de la culture ? Les phénomènes de type totémique ne sont pas l'expression d'une indifférenciation entre nature et culture; au contraire, 
ils nous rappellent que ces deux ordres entretiennent l'un avec l'autre des relations telles que les différences éprouvées dans le cadre culturel acquièrent une signification de la confrontation avec les différences perçues et répertoriées dans le monde naturel. En un autre langage, on dirait que le monde naturel a informe » le monde culturel. Pour LéviStrauss, le totémisme est une forme spécifique de notre propension à classer :

" (...) le prétendu totémisme n'est qu'un cas particulier du problème général des classifications et un exemple parmi d'autres du rôle fréquemment attribué aux termes spécifiques, pour élaborer une classification sociale" (1962a: 83).

Le totémisme, illusion (Lévi-Strauss) ou pure construction de l'anthropologie (Boas) ? Le débat n'ayant guère progressé depuis les années soixante, essayons d'aborder naïvement aux rivages de la côte Nord-Ouest. Il est difficile de dater avec précision les premiers emplois des termes " totem " et « totem pole " dans le contexte considéré. En tout cas, le premier de ces deux termes est bien attesté dans les plus anciennes monographies ethnographiques, de G. M. Dawson ( $c f$. Cote $\&$ Lockner, 1993) sur les Haida et de G. T. Emmons (1991) sur les Tlingit, qui datent des années 1870-1880. Le terme " totem " n'est jamais spécifiquement défini et renvoie tantôt à un groupe de parenté, tantôt à un emblème, il peut être indifféremment employé en place de "clan" (ou gens), ou de "blason ", ce dernier terme devenant l'équivalent de " sous-totem " dans la monographie d'Albert Niblack (1970 [1890] : 245-246) sur les Tlingit. Le terme " totem " en est venu à désigner aussi le guardian spirit individuel, l'animal protecteur des sociétés secrètes ou encore l'entité éponyme d'un groupe. A propos de l'emploi de " totem ", Goldenweiser (1910: 268) écrit : "Le terme est appliqué à l'entité éponyme, au nom lui-même [du groupe] et à la représentation symbolique de l'entité ". On le voit, le terme " totem " ne renvoie pas à un phénomène bien déterminé mais à un ensemble de phénomènes qui entretiennent entre eux des relations indéterminées, l'imprécision sur la nature de cette " chose " qu'est le " totem" ne pouvant qu'engendrer une imprécision comparable sur ce qu'est le " totémisme". 
Comme on l'a suggéré plus haut, les termes " totem " et " totem pole $"$, pour ce qui concerne la côte Nord-Ouest, sont inséparables. Les * mâts totem " ou " mâts totémiques "- ou encore " mâts héraldiques " (Lévi-Strauss, 1989 : 262) - sont, comme leur nom l'indique, des monuments faits d'un long tronc d'arbre décoré de figures sculptées et/ou peintes, imbriquées les unes dans les autres. A l'instar de certains poteaux de l'armature des maisons eux aussi décorés, les mâts témoignent de ce qui fonde la relation du groupe à son passé et rendent compte des statuts et des privilèges des familles et des individus qui les ont fait ériger (Halpin, 1984). De ces témoins désormais inanimés d'une culture autrefois prodigieusement riche, comme nous le rappellent les merveilleux clichés des années 1870-1880, Georges Duthuit (1974 [1946] : 315 ) livre une évocation qui rend bien compte du caractère fabuleux des figurations ${ }^{7}$ :

6 "Totem pole " est un terme générique; sous ce vocable, on distingue notamment les mâts frontaux (house poles), les mâts funéraires, les mâts commémoratifs érigés devant les maisons. Les premiers voyageurs et commerçants qui visitent la côte NordOuest à partir des années 1770 ont laissé des témoignages sur les mâts haida, tlingit et nootka. On doit à John Bartlett, qui se rend, en 1791, dans un village haida de l'île Langara, la première description d'un mât totémique. Bartlett en fit un croquis publié dans son journal de voyage. Cook a fait une description détaillée de poteaux de maison nootka (1778) : on peut se reporter à la gravure de John Webber qui accompagnait Cook lors de son troisième voyage. Pour un inventaire des premières descriptions de mâts, cf. Duff (1964), Chapman (1965) et MacDonald (1983). Marius Barbeau $(1939,1940)$ a soutenu l'hypothèse selon laquelle le mât totémique tel que nous le connaissons serait un objet de création récente, dont la fabrication se serait développée avec l'adoption par les Indiens d'outils en fer obtenus par la traite des fourrures, l'enrichissement des sociétés de la côte Nord-Ouest par le commerce s'étant traduit par un déploiement spectaculaire des signes de prestige.

7 En 1890, Boas faisait déjà remarquer que l'art de la côte Nord-Ouest n'était pas entièrement totémique, ou dirions-nous aujourd'hui, héraldique. Certains objets étaient sculptés dans des formes animales à des fins magiques. La représention d'un animal était liée à sa nature ou à ses attributs : ainsi un gourdin pour assommer les phoques était-il sculpté en forme d'épaulard, prédateur des phoques. D'autres objets, tels les plats cérémoniels utilisés lors des fêtes et des potlatch, reproduisaient la morphologie du phoque ou du lion de mer, car la graisse de ces animaux était un symbole de richesse. Les accessoires des chamanes sont décorés de sculptures qui représentent des animaux; il s'agit là non pas de blasons, mais de la figuration des esprits auxiliaires (loutre, chèvre de montagne) du chamane sous leur forme animale. Enfin, même si le nom de certains motifs de vannerie ou de tissage fait référence à des animaux, tel que " dents d'épaulard " ou " queue de corbeau ", ces motifs décoratifs ne sont en aucun cas héraldiques ( $c$. Boas, 1897a ; de Laguna, 1972 ; Emmons, 1991). 
" Hérissés de nageoires, de becs de rapaces, gonflées de narines carnassières et de paupières de batraciens, mêlant aux traits de l'homme ceux du corbeau, de la baleine-tueuse, de la lune et du soleil, ils servent d'arbres généalogiques aux familles régnantes, en mềme temps que de diplốmes d'honneur (...) n.

Le mât totem est devenu l'objet ou le monument emblématique - c'est le cas de le dire - des cultures de la côte Nord-Ouest, comme le suggèrent aux visiteurs les parcs publics et les alentours des musées. Autrefois, le mât totémique permettait d'identifier l'appartenance d'un individu à un groupe. Aujourd'hui, il résume une identité ethnique immédiatement appréhendable par les touristes, ainsi " le circuit des mâts totémiques " constitue-t-il l'itinéraire standard des croisières organisées entre Vancouver et Seward (sud-est de l'Alaska). Voyageurs et autochtones trouvent également leur compte dans cette désacralisation du mât totémique : les non-Indiens voient dans les mâts la manifestation la plus achevée de l'art de la côte Nord-Ouest ; les Indiens tirent une légitime fierté du fait qu'une forme d'expression artistique qui leur est propre en vienne à avoir valeur de symbole d'une région ${ }^{8}$.

Nous avons déjà indiqué que le totémisme n'est pas un thème central dans les travaux des premiers ethnologues de la côte Nord-Ouest, qui consistent principalement en monographies traitant de l'organisation socio-politique et du potlatch ou en recueils de mythes; quand la question du totémisme est abordée, elle l'est en général dans son rapport aux faits d'organisation sociale. Ainsi traite-t-on des relations entre le totémisme et la filiation, l'exogamie et la formation des groupes plutôt que des manifestations du totémisme en général. A partir de la conception la mieux partagée de l'hypothèse totémique, le totémisme de la côte Nord-Ouest apparaît aux analystes comme une version "faible ", voire altérée, d'un système interprétatif général dont la " théorie américaine *

8 Cf. Jonaitis (1989) et Tait in Halpin (1983). Les mâts de l'Alaska ont été sauvés et restaurés dans le cadre de la politique du New Deal de préservation de la culture indienne mise en cuvre dans les années 1930. Le Canada a adopté une politique similaire à partir des années 1950 ; retirés de leur site d'origine, les mâts ont été érigés dans des parcs nationaux ou placés dans les musées. 
donne cependant une présentation spécifique en l'associant à la transformation d'un totémisme individuel en un totémisme de clan.

A la base du totémisme, il y a l'idée, développée par l'Ecossais John McLennan (1869) ${ }^{9}$ d'une antériorité de la filiation matrilinéaire sur la filiation patrilinéaire, hypothèse sociologique qui ferait système avec la croyance attribuée aux sociétés " primitives " en vertu de laquelle la relation d'un groupe à une ascendance animale ou végétale s'établirait par le moyen d'une filiation utérine. L'un des résultats majeurs des analyses anciennes du totémisme est qu'il existe une relation nécessaire et suffisante entre l'identification d'un groupe de descendance à une espèce naturelle et l'existence de matri-clans exogames. Dans ses tout premiers travaux, Boas adopte cette position et inscrit son analyse de l'organisation sociale des $\mathrm{K}$ wakiutl dans le cadre de la théorie totémique en général ${ }^{10}$.

Dans son "First General Report on the Indians of British Columbia " (1889), Boas note l'existence de totems au sens de Frazer (1887) chez les peuples septentrionaux de la côte Nord-Ouest : Tlingit, Tsimshian et Heiltsuk. Les tribus sont subdivisées en phratries, chaque phratrie porte le nom d'un animal-totem ; à leur tour, les phratries sont subdivisées en gentes matrilinéaires. Dans les écrits qui suivent, Boas réserve l'utilisation de gens aux Kwakiutl dont, à la différence des Haida et des Tsimshian, qui sont clairement matrilinéaires, il juge que l'organisation sociale, à partir d'un substrat matrilinéaire, s'est infléchie

9 Adam Kuper (1988 : 83) remarque, non sans ironie, à la suite de R. A. Downie, biographe de Frazer, que le totémisme " comme le radar, le whisky et la marmelade, est une découverte ou une invention écossaise".

10 Il convient de remarquer que les premiers travaux sur la côte Nord-Ouest ont été publiés sous les auspices de la British Association for the Advancement of Science. Les recherches de Boas ont été initialement financécs par le British Anthropological Committee, placé sous l'autorité de E. B. Tylor. Boas inscrivait donc tout naturellement son propos dans le contexte d'une " orthodoxie " britannique selon laquelle les sociétés évoluent d'un régime matrilinéaire vers un régime patrilinéaire. Ce n'est que lorsque Boas obtiendra un poste à la Smithsonian Institution qu'il se dégagera de l'évolutionnisme (Kuper, $1988: 139$ ). 
vers un régime patrilinéaire ; pour les $\mathrm{K}$ wakiutl, en place de " totem ", il préfère employer le terme « blason " qui se rapporte à une manifestation mineure du totem.

Dans son importante monographie des Kwakiutl (1897b), Boas, tout en restant dans le langage de l'unilinéarité, inverse son point de vue initial et va à contre-courant des idées généralement admises alors. Il considère désormais que le système social des Kwakiutl a évolué de la patrilinéarité vers la matrilinéarité, notamment sous l'influence des tribus septentrionales (1897b : 334). En même temps, il renonce à l'emploi du terme gens pour lui préférer celui de "clan ", évocateur d'une orientation matrilinéaire de l'institution. L'hypothèse, qui sera pour un temps admise par J. Swanton (1904), de l'antériorité de la patrilinéarité sur la matrilinéarité, se fonde pour Boas sur plusieurs indices : le fils de la sœur ne succède pas à son oncle utérin, la résidence n'est jamais matrilocale, les clans ou communautés villageoises, à la différence des groupes matrilinéaires du Nord, sont constitués par la descendance patrilinéaire d'un ancêtre mâle. Les modalités de transmission des privilèges impliqueraient l'existence d'un double régime successoral, dont Boas ne formule pas encore clairement les principes. D'une part, certains privilèges - noms et blasons notamment - sont transmis en ligne paternelle selon la règle de primogéniture ; d'autre part, lors du mariage, "la femme apporte la position et les titres de son père en dot à son mari " qui les transmettra à son fils. Cette situation n'étant que la réitération d'une situation antérieure - le père de l'épouse ayant acquis ses titres par sa mère -, Boas considère comme tenable l'hypothèse qu'une règle de transmission utérine s'établisse par l'intermédiaire du mari (ibid. : 334-335). Boas note encore que des légendes kwakiutl sur l'origine des clans présentent certaines similarités avec les traditions des groupes septentrionaux. Il précise que les noms de clan peuvent relever de l'une ou l'autre des trois catégories suivantes : 1) nom dérivé de celui de l'ancêtre mythique ; 2) nom de lieu attaché à un territoire ; 3) nom honorifique rappelant un événement important de l'histoire du groupe (ibid. : 332-333). Les traditions se rapportant à l'acquisition des noms et des blasons empruntées par les $\mathrm{K}$ wakiutl aux groupes du Nord ou du moins marquées par l'influence septentrionale se répartissent en deux groupes : 1) celles pour lesquelles l'ancêtre revêt la forme d'un être surnaturel, propriétaire de privilèges, 
qui descend du ciel ou émerge du monde chthonien ou sous-marin ; 2) celles qui relatent la rencontre par l'ancêtre d'une entité surnaturelle qui lui accorde sa protection et la possibilité d'acquérir des biens symboliques qui seront transmis à l'ensemble du groupe (ibid. : 336-339). Ainsi, chez les Kwakiutl, l'entité représentée sous la forme matérielle du blason n'est-il pas l'ancêtre ${ }^{11}$. L'emblème du clan n'est l'objet ni d'un culte ni d'une prescription d'interdits. Boas remarque que seul un petit nombre de familles nobles, du fait de leur position généalogique par rapport à l'ancêtre, détient le droit de revendiquer l'usage des blasons et des privilèges.

On en arrive au célèbre article de 1920 dans lequel Boas reprend l'analyse de l'organisation sociale et des modes de transmission des biens chez les Kwakiutl et abandonne définitivement l'hypothèse totémique, ainsi que l'emploi, jugé fallacieux (1920 : 115), des termes gens et "clan ", au bénéfice du terme indigène numaym. Ici, le glissement sémantique l'emporte de beaucoup sur le glissement lexical. En effet, on ne saurait définir pleinement le numaym ${ }^{12}$ comme un groupe de descendance au sens strict du terme et donc faire du type de filiation un des critères de son identification. On pourrait plutôt définir le numaym comme une

11 Dans son compte rendu de l'ouvrage de Boas (1897b), Durkheim (1900: 337) écrit que chez les K wakiutl, "Le totem, c'est-à-dire l'objet qui sert de blason n'est plus l'ancêtre; ce n'est plus qu'un fétiche que l'ancêtre mythique a apporté à ses descendants ". Durkheim note que, dans ces conditions, "le totémisme kwakiutl est effacé $"$.

12 C'est dans une publication posthume (1966) que Boas nous livrera sa dernière conception du numaym, qu'il appelle désormais numayma. Le numayma n'est ni patrilinéaire, ni matrilinéaire. C'est une entité dont l'existence est indépendante des individus à travers lesquels elle se manifeste. Le numayma est constitué d'un certain nombre de sièges ou positions auxquels sont attachés un nom, un rang et des privilèges. Cette configuration de noms forme en quelque sorte le "squelette " du numayma. Les noms et les privilèges qui leur sont associés se transmettent soit par la filiation, soit par le mariage. Il arrive cependant que les titres de noblesse ne sortent pas de la famille aux fins de maximiser l'importance du patrimoine familial, objectif à quoi peuvent correspondre des cas d'endogamie du numayma. On en vient à considérer que les règles de transmission, si règles il y a, sont déterminées par l'objectif général de conservation et de renforcement d'un patrimoine symbolique. Il revient à Lévi-Strauss (1979) d'avoir développé, dans le sillage de Boas, une analyse novatrice de l'organisation sociale des $\mathrm{K}$ wakiutl à partir de la problématique de la "maison ", en s'inspirant des faits propres aux sociétés occidentales. 
unité sociale qui se donne avant tout comme un ensemble de positions hiérarchisées existant indépendamment des individus qui les occupent ; ces positions sont associées à des noms et à des privilèges qui sont transmis selon des règles complexes, en tout cas autres que celles qui sont censées régir le mode de filiation. A cette étape du développement de la pensée de Boas, mais on peut en dire autant de la pensée anthropologique dans son ensemble, il apparaît que le modèle associant totem, exogamie et matrilinéarité ne saurait résister à la confrontation avec quelque ethnographie particulière que ce soit. Boas constate que chez les Kwakiutl, les privilèges sont des biens individuels et en conséquence n'appartiennent pas au numaym en son entier, de telle sorte que le groupe de référence ne peut être considéré comme totémique (1920:125), à la différence de ce qu'il en va pour les sociétés septentrionales au sein desquelles chacun des membres du groupe de référence, quel que soit son statut, peut revendiquer l'usage du blason collectif. Chez les Kwakiutl, un enfant, à sa naissance, ne possède ni blason ni position au sein du groupe; il ne détiendra nom et blason que lorsqu'ils lui seront octroyés par un parent. Plus généralement, la notion d'une relation de l'ensemble du numaym à un animal de type totémique est inappropriée (ibrid. : 126). De même, l'identification d'un chef à un ancêtre animal ne relève pas d'un rapport direct fondé sur la croyance en une filiation de l'animal à l'homme mais d'un usage social de la métaphore. Enfin, Boas réfute l'hypothèse selon laquelle les faits kwakiutl relèveraient d'une forme résiduelle d'un totémisme ancien.

Dans le contexte des recherches sur la côte Nord-Ouest, un débat s'est instauré sur le point de savoir dans quel ordre sont apparus moitiés et lignages; il s'est cristallisé autour de deux questions distinctes dont les réponses s'excluent l'une l'autre : la moitié préexiste-t-elle au lignage ou est-elle le résultat de la fusion de plusieurs lignages ? Les traditions mytho-historiques rendent compte de la formation des groupes. Les Tlingit et les Haida rapportent qu'à un moment de leur histoire, la société était constituée de petits groupes locaux s'apparentant à des communautés villageoises. De migrations, de divisions ou au contraire de 
fusions des groupes initiaux ont résulté une forme d'organisation sociale fondée sur des regroupements locaux de clans, comme chez les Tsimshian, ou sur une dispersion sur l'ensemble du territoire de fractions claniques ayant conservé la mémoire d'une unicité initiale, du nom et du lieu d'origine du clan, comme chez les Tlingit et les Haida. E. Sapir (1965 [1915] se posera une question analogue : les moitiés sont-elles le résultat d'une fusion de lignages - il emploie le mot " clan " - ou au contraire les lignages qui composent les moitiés sont-ils issus d'une segmentation de moitiés en unités plus petites? E. Sapir penche pour la seconde hypothèse : 1) les moitiés sont présentes dans toutes les communautés villageoises ; 2) le blason principal de moitié est commun à l'ensemble des lignages qui composent la moitié. Au cours de l'histoire, des fractions se seraient dissociées du groupe principal et auraient acquis de nouveaux privilèges, tout en conservant une partie de leur patrimoine d'origine. Ainsi Sapir conclut-il : "On peut envisager que les phratries soient sociologiquement des formes réinterprétées d'unités tribales d'origine distincte" (1915 [1965] : 331). Sans prendre pour argent comptant - comme semble le faire Swanton - la valeur de vérité historique des mythes, tout porte à penser qu'il n'est pas possible d'aborder le problème de la formation des groupes si l'on n'en considère pas la dimension dynamique. Comme le souligne Federica de Laguna (1975 : 68-69), l'histoire des sociétés de la côte Nord-Ouest témoigne de mouvements constants de division et de fusion des unités sociales, aussi faut-il envisager le système moitié-lignage non pas comme statique, mais comme en constante recomposition, ainsi que l'auteur cité l'observe chez les Tlingit $(1952,1975)$.

Quelle est l'origine de la matrilinéarité sur la côte Nord-Ouest ? Cette nouvelle question en appelle immédiatement une autre : le système à blasons (crest system) est-il inhérent au système de moitiés et de lignages, et en conséquence les deux systèmes se sont-ils développés de façon concomitante ? On peut se demander sur cette lancée si l'existence de privilèges associés aux blasons renvoie principalement à la gestion des relations entre des groupes de parenté et à l'exploitation de l'espace, ou si, au contraire, on doit considérer que les groupes de descendance 
doivent leur spécificité à l'acquisition et à la transmission des blasons. L'ethnographie montre que matrilinéarité et bilinéarité offrent deux types de réponse à la question du contrôle de la transmission des biens symboliques.

Pour ce qui concerne l'origine géographique de la diffusion ${ }^{13}$ de la matrilinéarité, bon nombre d'ethnologues, à l'instar de Swanton (1905b : 670-671), ont soutenu que les institutions matrilinéaires se seraient développées à partir d'un foyer qui se situerait dans la région des rivières Nass et Skeena (Colombie britannique) ; il y aurait eu ensuite diffusion en direction du sud de la côte Nord-Ouest, mais aussi en direction des groupes athapaskan de l'intérieur. L'hypothèse d'un cheminement inverse, de l'intérieur vers la côte, serait peu vraisemblable. Il semble en effet que l'essor du système à blasons ne soit pas séparable du spectaculaire essor du cérémonialisme de la côte Nord-Ouest ; en outre, il paraît certain que la validation des privilèges par des dons n'a pu intervenir que dans un contexte d'exploitation d'un environnement naturel d'une richesse exceptionnelle, et non au sein de petits groupes de chasseurs-cueilleurs, ce qui n'empêche pas de penser que l'émigration de groupes de l'intérieur vers la côte, qui s'est traduite par une agrégation de nouveaux venus à des groupes déjà en place, tels les Tlingit et les Tsimshian, a pu stimuler le développement du système à blasons et à clans. De Laguna (1975), à l'issue d'un tour d'horizon comparatif des sociétés de la côte Nord-Ouest, conteste cette vision trop simple des choses pour affirmer que les groupes matrilinéaires exogames étaient très répandus dans le Nord-Ouest américain. Leur existence aurait précédé la migration vers la côte de groupes d'origine Nadéné, à savoir les Tlingit et les Haida. Certes, les embouchures des fleuves Nass et Skeena ont été de véritables lieux de brassage de populations venues d'horizons

13 On peut se reporter à l'étude de F. de Laguna (1975) qui reprend l'ensemble du débat sur l'origine de la matrilinéarité dans la région. L'auteur analyse les institutions matrilinéaires en fonction du nom des moitiés, des blasons qu elles détiennent et de leurs traditions légendaires et historiques. Outre une origine que l'on pourrait qualifier de régionale, certains ont défendu la thèse d'une origine asiatique, ainsi Marius Barbeau, qui, à travers l'analyse de la mythologie tsimshian, croit découvrir les preuves d'une émigration à partir d'un foyer qui serait situé en Sibérie, avec les îles Aléoutes pour région de transition ( $f$. Duff, 1964). 
différents; à ce titre, la région a joué un rôle important dans la formation des groupes actuels, mais ces indications ne sont pas suffisantes pour en faire le berceau de la matrilinéarité sur la côte Nord-Ouest. F. de Laguna rappelle que bon nombre de blasons ont une origine intérieure et non côtière, à commencer par le fameux Corbeau qui serait une création des Athapaskan occidentaux (Chowning, 1962). Dans ce débat, Barbeau ( $f f$. Duff, 1964) intervient de manière plus que discutable, en avançant que l'exogamie des moitiés et le totémisme auraient connu leur fortune à la suite du contact avec les Européens, de telle sorte que le caractère totémique des moitiés et des lignages aurait trouvé sa pleine expression pendant la période coloniale, à partir des années 1860.

La question de l'origine de la forme de totémisme propre à la côte Nord-Ouest a fait l'objet d'une hypothèse initialement formulée par Boas et qui a notamment été reprise par Charles Hill-Tout (1905) et Swanton (1905a). Boas $(1897$ : 332, 336, 662) remarque que les guardian spirits ou esprits gardiens coexistent avec les totems de clan. Les guardian spirits, que Boas appelle " manitous individuels" (1897b, 1898, 1916b), seraient à l'origine des totems : le totem de clan est l'esprit gardien de l'ancêtre devenu celui de sa descendance, les descendants respectant l'espèce animale ou végétale par laquelle l'esprit s'est manifesté. Pour Hill-Tout, l'esprit gardien individuel, l'animal tutélaire qui préside aux destinées des sociétés secrètes et le totem de clan ne feraient qu'un (Goldenweiser, 1910 : 268). Si Boas et Swanton envisagent le totémisme comme un phénomène à la fois religieux et social, ils ne considèrent cependant pas, à la différence de Hill-Tout, que la relation de filiation entre animal totémique et clan soit nécessaire. Boas a sur ce point une formulation assez précise :

" (...) ce que nous appelons totémisme [c'est] une forme d'organisation sociale au sein de laquelle certains groupes sociaux considèrent avoir une relation de parenté surnaturelle avec certaines espèces d'animaux ou certaines classes d'objets" (1910a [1974] : 246).

Ainsi, pour Boas, les idées religieuses qui se rapportent au totémisme font référence à la relation personnelle d'un homme avec certaines 


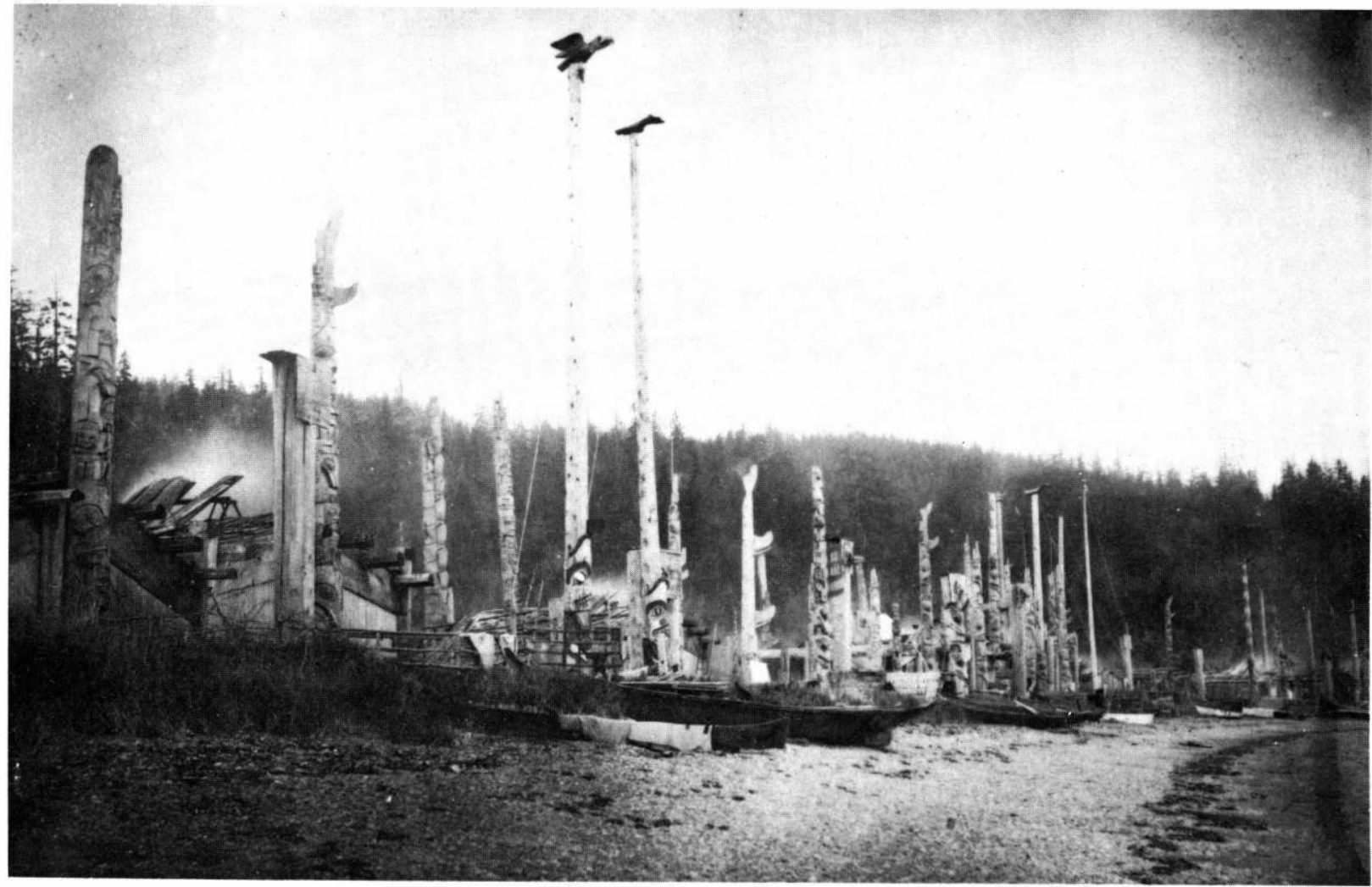

Vue du village hä̈da de Skidegate. Au premier plan, forêt de mâts héraldiques.

(Cliché Richard Maynard, 1994. Par courtoisie du Royal British Columbia Museum, $n^{\circ}$ PN 9078, Canada) 


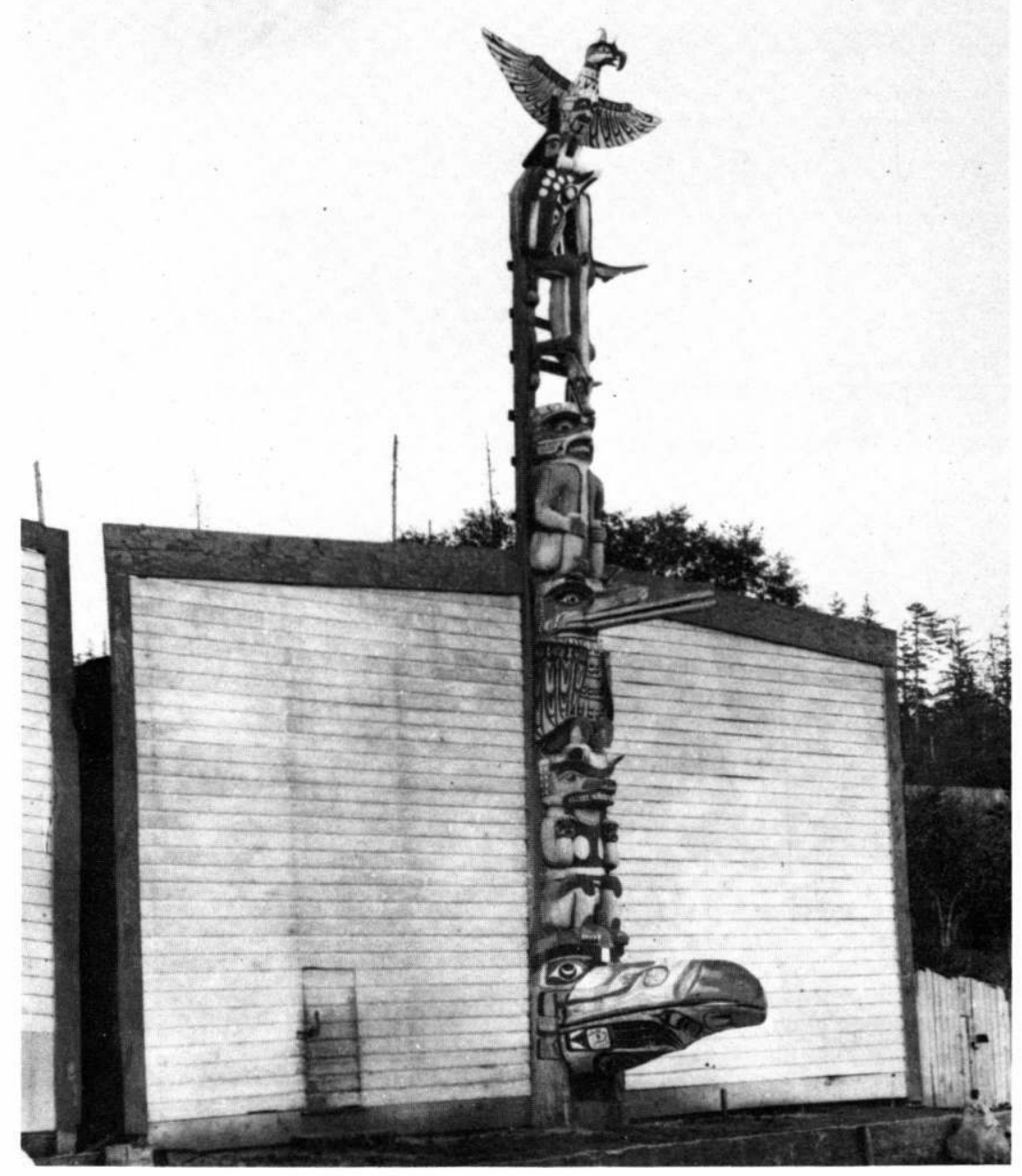

Village kwakiutl d'Alert Bay.

Poteau érigé devant la maison du chef Wakas.

(Cliché Edgar Fleming, 1896. Par courtoisie du Royal

British Columbia Museum, $n^{\circ}$ PN 12217, Canada) 
catégories d'esprits surnaturels, et ce qui caractérise le totémisme, c'est l'association de certains types de pouvoirs surnaturels avec des groupes sociaux" (ibid. : 251).

La notion de totémisme individuel rend compte d'une étroite relation de protection associant généralement un animal et un individu, cette protection se manifestant dans une délégation ou un transfert de pouvoirs de l'animal protecteur à l'individu protégé. Dès lors, le passage d'un totémisme individuel à un totémisme collectif s'opère par le moyen d'un changement d'échelle de l'intervention de l'esprit gardien : d'une relation entre deux êtres singuliers - un animal, un homme -, nous passons à une relation entre un être individuel et un être collectif, qui procède de l'appropriation par un groupe d'une espèce naturelle, dont la figuration blasonnée devient emblématique du groupe. Dans le langage de Boas (1916b : 532), il y a " socialisation " de l'esprit gardien. C'est à partir d'une référence commune au rôle joué par l'esprit gardien que Boas (Boas, 1897b : 393-394 ; 1899 [1974] : 68), pour les Kwakiutl, établit un parallèle entre l'origine des clans et celle des sociétés secrètes.

La " théorie américaine " de l'antériorité du totémisme individuel sur le totémisme collectif a été critiquée par Durkheim (1912) et par Frazer $(1922)^{14}$. Frazer, notamment, en souligne les limites, précisément parce que cette théorie est fondée uniquement sur des faits nord-américains et donc ne peut avoir une portée générale. Il se demande comment expliquer la corrélation entre totémisme et filiation matrilinéaire, compte tenu notamment que les travaux disponibles sont muets sur l'existence des esprits gardiens des femmes. A la différence de Hill-Tout, Boas (1910: $392,1916 \mathrm{~b}: 515-516)$ ne prétend pas que la " théorie américaine " puisse être généralisable à l'ensemble des sociétés dites totémiques, simplement parce que, conformément à la position qu'il a toujours défendue, les faits ethnographiques, à ses yeux, doivent être systématiquement replacés dans leur contexte géographique et historique.

14 Je donne la référence française publiée en 1922 sous le titre Les origines de la famille et du clan. Pour l'original, se reporter à Totemism and Exogamy, 1910. Cf. aussi Durkheim, 1912 : 246 sq. Le débat est résumé in Davy, 1922 : 251 sq ; ff. aussi Van Gennep, 1920. 


\section{Le système à blasons}

Un blason est un marqueur identitaire appartenant à un patrimoine symbolique détenu par un groupe - moitié, clan ou lignage - et dont le droit d'usage est exercé par des personnes représentatives des groupes détenteurs ${ }^{15}$. La détention d'un blason est le résultat soit d'une transmission au sein d'un groupe en ligne matrilinéaire, soit d'une acquisition faite dans des circonstances déterminées, telles que la guerre, l'échange, etc. L'élément d'identification sociale qu'est le blason est établi à partir de la personnification d'une entité du monde naturel ou surnaturel. Il s'agit très généralement d'un animal, mais dans certains cas le nom du blason peut renvoyer à une plante, un site, un phénomène naturel (Eboulis), une manifestation météorologique (Nuage, Neige, Arc-enCiel), un élément du cosmos (Lune, Etoiles), un objet usuel, un être monstrueux tel que Wasgo dit "Ours de mer "16. Il existe des blasons principaux qui se sont diversifiés en des blasons subordonnés dont la distribution renvoie à une hiérarchie de rangs. Le propre de ces blasons " particularisés" est que leur nom et les représentations qui leur sont associées évoquent un aspect particulier du blason " générique " initial ${ }^{17}$. Ainsi, chez les Tsimshian, le blason générique Corbeau peut se manifester à travers " Corbeau céleste ", "Corbeau de cuivre ", "Corbeau de nacre ", "Corbeau mangeur de foie de saumon ", etc. (Halpin, 1973, 1984 : 24). Chez les Tlingit et les Haida, pour marquer la différence entre diverses occurrences d'un même blason, sans que soit en cause un système hiérarchique, l'animal-blason peut être identifié par son habitat (repaire, nid, etc.) ou par une caractéristique morphologique (nageoire, aile, os), ainsi, pour faire retour à Corbeau, aurons-nous, par exemple, les blasons "Nid de Corbeau ", "Os de Corbeau " ( $c f$. de Laguna, 1972 : 452 454), etc.

15 On peut se reporter aux définitions données par Halpin $(1973: 27,1984: 17)$, Boelscher (1988 : 142), Kan (1989 : 69).

16 Chez les Gitksan, par exemple, les blasons se répartissent ainsi : animaux : $63,8 \%$; plantes : $2 \%$; phénomènes naturels : $2,8 \%$; objets inanimés : $10,7 \%$; humains : 20,6\% (Cove, $1987: 113$ ).

17 On doit à Marjorie Halpin (1984 : 24) la distinction entre blason " générique " ou generalized crest et blason " particularisé " ou particularized crest. 
Les blasons les plus importants renvoient à une espèce animale. Cependant, les animaux-blasons ne forment ni une famille empirique associant des animaux relevant d'un même " usage " pratique, ni une famille zoologique, que l'on envisage les ensembles d'animaux-blasons au niveau de la moitié ou à celui du lignage. Parmi ces animaux emblématiques, on a des mammifères terrestres et marins, des insectes, des poissons, des mollusques, etc. Certains de ces animaux sont comestibles, d'autres pas ; certains sont chassés, d'autres pas ; certains sont propres à satisfaire des besoins économiques ou techniques, d'autres pas ${ }^{18}$.

D’une manière générale, les sociétés de la côte Nord-Ouest n'observent aucun interdit vis-à-vis de l'animal-blason, à quelques exceptions près cependant, telle celle de l'Epaulard (Swanton, 1905a; Boas, 1916b : 500), quoique dans ce cas particulier, l'interdit renvoie non pas à la relation entre le groupe et son animal-blason, mais à la croyance qu'ont les chasseurs de mammifères marins qu'après leur mort ils deviendront des épaulards (Goldenweiser, 1910). Les Tlingit n'interdisent pas de tuer l'animal-blason, mais ils observent une attitude de respect à son égard et revendiquent pour eux-mêmes les qualités attribuées à l'animal ; toujours chez les Tlingit, si l'animal-blason s'est rendu coupable d'avoir blessé un membre de la moitié opposée, celle-ci peut faire l'objet d'une demande de compensation émanant de la moitié " victime " (de Laguna, 1972 : 825-826, Kan, $1989: 68)^{19}$.

Il faut introduire ici une précision importante sur l'animal-blason dont le caractère précisément " animal " est ambigu. Les animaux ont en effet une nature duelle. Ce sont certes des animaux bien réels, qui peuvent être éventuellement chassés et mangés, mais, comme la mythologie nous le rappelle, ils possèdent concurremment un " aspect " qui les apparente aux humains, dont ils comprennent la langue : les animaux vivent dans des villages, ils sont organisés en société, etc. Dans un passé lointain, les animaux étaient anthropomorphes, et ce n'est que lorsqu'ils

18 Chez les Tlingit et les Haida, par exemple, les corbeaux et les aigles sont chassés mais ne sont pas mangés, leur chair étant jugée inconsommable (Oberg, 1973 : 47 ; Boas, $1916 \mathrm{~b}: 500$ ). L'ours noir est considéré comme un animal comestible tandis que l'ours brun est chassé pour sa fourrure (Oberg, ibid.).

${ }^{19} \mathrm{Cf}$. aussi Cove, $1987: 123-124$ pour les Gitksan. 
revêtaient leur corps, qui de poils, qui d'écailles ou de plumes, qu'ils apparaissaient tels que nous les connaissons. C'est cette capacité des animaux de passer d'une forme à une autre que rappelle une sculpture héraldique associant dans la figuration des mêmes " êtres " des éléments animaux et humains, des becs, des plumes, de la fourrure, mais aussi des mains, des pieds, etc. Comme le note de manière très pertinente MarieFrançoise Guédon (1984 : 142) : “ La transformation n'est pas tant un processus qu'une qualité correspondant à de multiples identités ou de multiples points de vue ou réalités que l'on attribue à une entité particulière ". La différence entre les humains et les autres êtres naturels réside principalement en ceci que les humains n'ont pas la capacité innée d'être source de pouvoirs, de telle manière qu'ils ne peuvent détenir de tels pouvoirs qu'en établissant des relations ad boc avec le monde surnaturel (Boelscher, 1988 : 172 sq ; Guédon, 1984 ; Halpin, 1973 ; Swanton, 1905 ; Cove, 1987).

L'existence d'un blason peut rendre compte d'une modalité de la localisation du groupe dans l'espace. Chez les Tlingit, on a une catégorie de blasons que l'on peut appeler " emblèmes de terre " (landmark crests), qui font référence à un territoire effectivement occupé ou à tout ou partie d'un territoire ancien. Les blasons renvoient souvent aux particularités d'un paysage, d'un cours d'eau, d'un site : baie, montagne, ou glacier, dont les dénominations peuvent être à l'origine de noms de clans. Cette emblématisation de l'espace, pour autant qu'elle se manifeste par un récit relatant l'histoire de la relation des ancêtres à un lieu, vaut légitimation d'une revendication du clan sur ce lieu. Ce type de blason n'évoque pas une territorialité mythique, mais rend compte de l'histoire de l'appropriation d'un espace. Des blasons tels que Mont Saint Elias, Ahrnklin River ou Copper River sont représentés sur des pièces de vêtement ou certains objets tels que des pipes; ils sont également peints sur les visages (de Laguna, 1972 : 455, 1975 : 91) ${ }^{20}$. Parmi les objetsblasons remarquables, on mentionnera encore le très caractéristique chapeau tressé, surmonté d'une colonne de disques en vannerie (Halpin,

20 On peut se reporter aux superbes planches ( $L$ et IL) illustrées de l'ouvrage de Swanton (1908). 
1984 : 27), dont le nombre - de trois à neuf - signalait l'importance statutaire du porteur de ce couvre-chef. Autres objets-blasons notoires pour les Tlingit : les cloisons (Cloison-Corbeau ou Cloison-Aigle Royal) et les poteaux de maison (Poteau-Ours ou Poteau-Aigle) (de Laguna, 1972 : 315-326) dont les motifs restituent des épisodes mythiques (de Laguna, ibid. : 455).

Parler de la "valeur" des blasons, de la place plus ou moins grande qui leur était assignée dans l'imaginaire ou la symbolique de la côte NordOuest autorise à introduire la notion de blason secondaire pour désigner les emblèmes qui ne sont pas d'origine mythique ou mytho-historique, mais portent au contraire témoignage d'événements intervenus dans la vie récente des groupes. On a un bel exemple de création d'un blason secondaire avec l'adoption par les Kagwantan (moitié Aigle des Tlingit) de l'aigle américain au titre d'insigne des officiers de la marine, dont les intéressés tirèrent un prestige suffisant pour qu'à l'occasion d'un potlatch ils s'autorisent à accueillir dans leur parentèle les marins en poste à Sitka (Alaska) (de Laguna, 1972 : 450). On peut évoquer aussi l'exemple d'un chef haida dont la présence à un potlatch était très attendue et qui apparut en exhibant un collier de grenouilles vivantes attachées par les pattes : en souvenir de cet événement mémorable, la famille de ce chef s'est octroyé le blason Grenouille (Boelscher, 1988 : 147). Voici enfin un grand panier utilisé à l'occasion de plusieurs potlatch : il fut promu au rang d'emblème sous le nom de Mère-Panier (Oberg, 1973 : 44-45).

Un blason ne consiste pas seulement en une représentation plastique (sculpture, peinture), il peut prendre la forme d'une expression du type chant, danse, voire cri cérémoniel, mimique, etc ${ }^{21}$. La figuration du blason est reproduite sur de multiples supports : canot, pagaie, tambour, coffre, louche, cloison et poteau de maison, mât, chapeau, coiffure (beaddress), heaume, masque ou autre objet cérémoniel ${ }^{22}$. Le blason est

21 Quand un guerrier tlingit mourait au combat, il poussait le cri de l'animalblason représenté sur son heaume (Kan, 1989 : 340).

22 Halpin (1984: 21) a établi une liste d'objets que les Tsimshian ne considèrent pas comme héraldiques (crest-bearing objects) : masques, hameçons, Cuivres, cuillers, couvertures, tabliers de danse. Il semble quil existe des différences importantes parmi les sociétés de la côte Nord-Ouest quant à la nature aliénable des blasons. Le Cuivre, qui pouvait être échangé entre des lignages, était considéré comme un objet aliénable, 
également rappelé par des tatouages de corps (bras, poignets, mains, doigts) et de visage ( $f$. Swanton, 1908). Le blason ne s'appréhende cependant pas à travers plusieurs figurations à la fois ; au contraire, un blason est généralement associé à une forme particulière de figuration ou d'expression. Chez les Teqwedi de la moitié Aigle des Tlingit de Yakutat, le blason Ours est figuré sur un plat cérémoniel ; chez les Nanyaayi, le même blason sera représenté par une simple peau d'ours ${ }^{23}$. Chez les Cankuqedi et les Kagwantan de la mệme moitié tlingit Aigle, le blason Epaulard est figuré sur les heaumes, alors qu'il est représenté, chez les Teqwedi, sur la canne du maître de chant, les tambours et les chapeaux (de Laguna, 1972 : 452). Ces distinctions sont autant de rappels des conditions d'" invention " ou d'acquisition du blason. Contrairement à ce qu'il est généralement admis, le blason n'est pas seulement l'image matérialisée d'un animal-blason, c'est aussi et surtout un ensemble de privilèges acquis et transmissibles. En ce sens, comme le remarque Sapir (1965 [1915] : 329), il est plus correct de dire, par exemple, qu'un chef possède le droit de peindre l'emblème Oiseau-Tonnerre sur le fronton de sa maison que de dire qu'il est propriétaire du blason Oiseau-Tonnerre.

Considérons maintenant le lexique indigène pour mieux cerner la nature des blasons et leur fonction dans le système social, en remarquant que si les analystes ont pu commettre des confusions terminologiques, elles tiennent aux multiples sens que recèlent les termes descriptifs euxmêmes. Chez les Haida, le terme gwaay gaang désigne à la fois le lignage et le blason, en tant que source de privilèges (Boelscher, 1988 : 142), ce qui signifie que l'identité du lignage s'apprécie à l'aune des privilèges qu'il détient. Les Tsimshian disposent des termes ptex, ayuks et $d z e p k$ pour

ce qui, selon l'auteur cité, est contraire à la conception mêrne du blason. Kan (1989 : $246,344)$ considère que les Cuivres tlingit sont des objets héraldiques, dont la valeur est cependant moins importante que celle d'autres objets. En échangeant les Cuivres, les nobles tlingit violaient les règles qui interdisaient la cession de biens héraldiques en dehors de la moitié et du clan.

${ }^{23}$ Chez les Tlingit, une tradition rapporte que l'acquisition de ce blason s'est faite à l'occasion du mariage d'un individu et d'une ourse ; une tradition rapporte que lors du Déluge, les gens du clan Nanyaayi trouvèrent refuge au sommet d'une montagne en compagnie d'un ours grizzli et d'une chèvre de montagne; depuis, ils ont pour emblème principal la peau d'ours et comme emblème secondaire la tête de chèvre de montagne (Swanton, 1908 : 415-416). 
désigner le "blason ", mais ptex désigne aussi le clan au titre de groupe à blasons (crest group). Ce même terme désigne aussi l'entité totémique à partir de laquelle sont générés plusieurs blasons particuliers. L'utilisation d'un même terme pour définir à la fois le clan et l'animal totémique est, selon Halpin (1973 : 114-115), "l'expression d'une association symbolique étroite entre le clan et l'animal ". Alors que ptex ${ }^{24}$ fait référence à l'espèce animale dont les principaux blasons sont issus, ayuks en désigne les dérivations symboliques à l'intérieur de l'espèce (Loup blanc, Grenouille géante, Corbeau dédoublé). Une douzaine d'ayuks peuvent être associés à un seul ptex ${ }^{25}$. Ayuks ${ }^{26}$ est aussi le blason détenu par les clans et les maisons. Les Tsimshian utilisent avec $d z e p k$ un terme qui fait référence à la représentation matérielle du blason. Selon Sapir, ce terme signifie " ce qui est fait " ou " ce qui est représenté sous sa forme visible " (cité in Halpin, 1973 : 114 ; cf. aussi Cove, 1987 et Guédon, 1984). Pour résumer, on peut dire que ptex renvoie au concept de blason, dzepk à l'image et ayuks à l'objet, c'est-à-dire à la fois au support et à l'image qui matérialise le privilège qui lui est associé27.

A ces trois déterminations sémantiques, la terminologie tlingit, en ajoute une quatrième, associée aux idées d'ancestralité et d'histoire. A la notion de blason correspondent trois termes distincts : shagoon, sbuka et at' $u$ (at' $u$ ? $u$ ou at'oow) dont la définition qu'on peut en donner ne va pas sans poser quelques problèmes, car les champs sémantiques concernés dans chaque cas interfèrent largement les uns par rapport aux autres, du moins si les ethnologues ont bien percé les significations des termes cités. Shagoon est un terme polysémique; il renvoie à l'origine, à l'héritage

${ }^{24}$ Le terme ne peut être employé pour désigner le blason d'une maison; son usage est réservé à la désignation du blason du clan.

25 Selon Halpin (1973 : 117), chaque clan tsimshian est associé à deux animauxblasons, à partir desquels sont générés des blasons particuliers ; ainsi, huit animauxblasons sont au fondement du système à blasons tsimshian.

26 Ayuks signific " mettre" (to put on). Le terme, selon Cove (1987: 115), contiendrait l'idée que de même qu'un vêtement transforme la personne qui le porte, le blason figuré et donc visible transforme l'objet ordinaire en objet surnaturel.

27 M.-F. Guédon, communication personnelle, octobre 1997. En kwakw'ala, le terme "blason" désigne principalement une sculpture (Sapir, 1915 [1965] : 329). Notons que le terme $k$ ikw signifie à la fois "mât totémique " ou " pièce sculptée" (McGrubb, 1977 : 183) 
ancestral et au destin d'un groupe ou d'un individu ${ }^{28}$ tels qu'ils sont déterminés par les ancêtres ou le totem du clan (de Laguna, 1972 : 455, 813) ; shagoon signifie plus particulièrement "ancêtre " dans les deux sens d'ancêtre mythique et humain, alors que shuka désigne l'ancêtre en général, avec l'idée d'un ancêtre d'avant, d'un ancêtre "prédécesseur " (Dauenhauer et Dauenhauer, 1994 : 20). Ce même terme shagoon est utilisé par les Tlingit pour nommer à la fois l'ancêtre et l'animal-blason (de Laguna, ibid.; Kan, 1989 : 68, 69), ce qui ne veut cependant pas dire qu'il y a confusion entre les deux significations : dans certains cas, ancêtre et animal-blason tendent à se confondre l'un avec l'autre, dans d'autres cas, le terme désignera seulement l'animal-blason et non l'ancêtre (de Laguna, 1972 : 455-456, 833-834). A l'instar des Tsimshian, les Tlingit établissent une différence entre shagoon, entité à partir de laquelle sont dérivés des privilèges légitimés par un mythe, et at' $u$, symbole de cette entité (de Laguna, 1972 : 455 ; Oberg, 1974 : 44) en tant qu'elle représente des privilèges. Le terme tlingit at' $u$ est équivalent au terme tsimshian ayuks. Une analyse récente du terme at' $u$ révèle qu'il signifie littéralement " une chose ou un objet possédé ou " acheté ". $A t$ 'u s'applique à une grande variété d' "objets" : un territoire symbolisé par une montagne ou un glacier, un esprit, un nom individuel, un motif plastique, un chant ou encore la représentation sur un vêtement d'un épisode du grand mythe de Corbeau (Dauenhauer et Dauenhauer, 1994 : 15). Enfin, sbuka désigne l'image ou le motif qui représente le blason (Dauenhauer et Dauenhauer, ibid. : 20). L'analyse de ces termes montre très clairement que l'identité d'un groupe est organiquement liée, non pas à son nom, mais aux blasons qu'il détient. L'identité n'est donc pas fondée sur l'être mais sur l'avoir, c'est-à-dire sur des détentions matérielles et symboliques. Leonard Adam

${ }^{28}$ Il semblerait que chez les Tlingit la notion de destin individuel soit intimement liée au droit d'usage de certains blasons dont on pensait qu'ils exerçaient une influence sur la personne. On symbolisait cette relation en frottant la bouche d'un enfant avec une pierre gravée dont le motif représentait le blason de son groupe (Kan, $1989: 69$ ). 
(1913 : 250) ${ }^{29}$ ne disait pas autre chose dans son étude sur l'organisation sociale des Haida et des Tsimshian :

" [les blasons] n'ont rien à voir avec ce que le groupe 'est' mais seulement avec ce qu'il 'a'. Les membres d'un groupe totémique de type dégénéré (degenerate) ne disent pas 'Nous sommes différents de vous autres', mais 'Nous possédons quelque chose de différent par rapport à vous !' et dès lors les emblèmes sont évalués les uns par rapport aux autres, 'Nous avons plus que vous!' Et rapidement un processus est à l'œuvre par lequel les blasons deviennent des emblèmes du rang social, même des biens qui peuvent être échangés contre d'autres. „30

D'une manière générale, le blason principal de la moitié correspond au nom de la moitié, comme chez les Tlingit, où Loup et Corbeau sont des noms de moitiés et de blasons; chez les Haida en revanche, si Aigle est le blason majeur de la moitié Aigle, c'est Epaulard qui est le blason de la moitié Corbeau (Sapir, 1915 [1965] : 326-327 ; Swanton, 1905a : 107). Sur une douzaine de blasons que possède chaque lignage, un certain nombre sont communs à plusieurs lignages d'une même moitié : le blason Ours Grizzli est partagé par douze lignages des Corbeaux haida, le blason Castor par une douzaine de lignages des Aigles et Epaulard par sept autres de cette même moitié ${ }^{31}$. Prenons l'exemple de la communauté haida de Masset, dont les habitants sont connus sous le nom de GaxaXadee. Le village réunit des membres des moitiés Corbeau et Aigle.

29 Partant d'un point de vue évolutionniste, Adam se rallie à la thèse dégénérationniste qui associe l'altération du totémisme de la côte Nord-Ouest à sa désacralisation.

${ }^{30} \mathrm{Je}$ traduis ici la citation à partir de la traduction qu'en a faite Mariane Boelscher de l'allemand en anglais (Boelscher, $1988: 151$ ).

${ }^{31}$ A titre d'exemple, voici la liste des emblèmes des lignages de la moitié Corbeau des Haida : Epaulard, Lune (d'origine tsimshian), Ours Grizzli, Arc-en-ciel, ChapeauLion de mer, Oiseau-Tonnerre, Nuages, Roussette, Loup, Flicker (sur un chapeau), Corbeau, Faucon, Arbre, Nageoire de Corbeau, Grizzli de mer, Ours noir, Belette, Hibou, Raie, Chèvre de montagne (d'origine tsimshian), Bois flotté ou Celui qui marche avec la marée (qui porte le nom de Tsamos, représenté sous la forme d'un Lion de mer ou d'un Epaulard), et Enfant-de-Femme-Propriété. Chez les Corbeaux, les blasons communs au plus grand nombre de lignages sont : Epaulard (partagé par tous, car Epaulard est le blason de moitié), Ours Grizzli, Arc-en-ciel et Celui qui marche avec la marée ; chez les Aigles, ce sont Aigle, Castor (d'origine tsimshian) et Callyonime (poisson) ( $f$. Swanton, 1905a : 108-117 ; 268-276). 
Epaulard, blason principal des Corbeaux, est commun à l'ensemble des lignages alors que Roussette n'est détenu que par deux lignages et OiseauTonnerre par un seul. Pour les Aigles, l'emblème de la moitié du même nom est partagé par l'ensemble des lignages, Castor est commun à quatre lignages ( $c f$. Swanton, 1905a ; Boelscher, $1988: 34,141,148-149$; de Laguna, $1975: 62-63)$. Il est admis par les analystes qu'il y a homologie entre l'ancienneté d'un blason et l'importance de son assise, ou qu'à l'inverse, une assise minimale signale un blason récent. Quand un blason est d'origine récente, son usage est restreint à un lignage ou à une maison (Boas, 1916b ; Goldenweiser, 1910; Sapir, 1915). Fidèle à ses conceptions évolutionnistes, Swanton a cru pouvoir faire l'hypothèse qu'entre blasons anciens et blasons récents il y avait déperdition du caractère " totémique " de ces emblèmes et passage d'un héraldisme sacré à un héraldisme profane. Certes, certains blasons, comme on l'a vu, ont une origine accidentelle et relativement récente, qui est sans rapport avec les exploits des ancêtres; cependant, l'hypothèse de Swanton est mise à mal par les faits : les blasons Ours Grizzli et Castor des Haida sont des blasons anciens, mais d'origine tsimshian ${ }^{32}$ : ils ont fait l'objet d'un échange avec les Haida au titre de biens matériels de prestige et non de biens sacrés.

La transmission en ligne maternelle n'épuise pas les modalités de la circulation des blasons. Chez les Haida et les Tlingit ${ }^{33}$, certains blasons peuvent être prêtés aux enfants par le lignage ou le clan du père. Dans la société tlingit, les blasons de clan - notamment les objets-blasons -, sont considérés comme aliénables et comptent de ce fait parmi les types de

${ }^{32}$ En outre, selon Swanton (1905: 65-66) et Boas (1916b), les Tlingit, les Haida et les Tsimshian, au cours de leur histoire, ont échangé des blasons. Certains blasons des Corbeaux haida, tels qu'Ours Grizzli, Chèvre de montagne, Lune, Arc-en-ciel, seraient d'origine tsimshian. Barbeau (1929: 172) souligne que les blasons auraient une valeur de témoignages commémoratifs d'expériences surnaturelles vécues par des ancêtres dont les circonstances et les conséquences sont rapportées dans les mythes. Certains blasons célèbrent des événements pseudo-historiques et ont donc un caractère profane.

${ }^{33}$ Chez les Tlingit, le prêt de blason par un père à son fils est considéré comme l'expression d'une relation de bonne entente entre les deux parents. On cite le cas d'un homme qui, pour montrer son attachement à son enfant mort, lors de la veillée funéraire, déposa à côté du cadavre un objet héraldique appartenant à son clan (Kan, $1989: 153)$. 
biens échangés dans le cadre des prestations matrimoniales, alors qu'en milieu tsimshian, au contraire, les blasons ne sont ni échangés ni vendus à l'occasion des mariages. Chez les Tsimshian précisément, si les blasons sont rarement donnés, en revanche, ils sont prêtés à l'intérieur du clan en témoignage de solidarité avec les partenaires emprunteurs, ou dans un geste de générosité fait à leur endroit.

Des blasons peuvent être acquis au titre de butin de guerre ${ }^{34}$, en compensation d'un crime, en témoignage de gratitude pour un service rendu ; ils peuvent être échangés lors de potlatch ou encore être accaparés à la suite de l'extinction d'une famille. Chez les Tsimshian, les blasons pris au cours d'un combat sont montrés publiquement en signe de victoire jusqu'à ce qu'ils soient rachetés par les vaincus. S'approprier un blason appartenant à un autre clan en en faisant sculpter ou fabriquer la représentation matérielle sans l'accord du clan propriétaire est considéré par ce dernier comme insultant. Parfois, un tel coup de force équivaut à une demande de rançon, la partie lésée devant alors verser une compensation à la fois pour laver l'affront qu'elle a subi et réinvestir son droit sur le blason (cf. Barbeau, 1929 ; Halpin, 1984 ; de Laguna, 1972 : 458-461 ; Swanton, 1905a, 1908).

Le droit à l'utilisation d'un blason par un chef ou un personnage de haut rang doit être validé à l'occasion d'une distribution de biens à laquelle sont invités les représentants de la moitié opposée qui sont récompensés pour le service rendu à l'unité invitante. Au cours de la cérémonie, le blason est non seulement montré de manière ostentatoire, mais encore son exhibition publique est toujours accompagnée du récit de son acquisition par l'ancêtre du groupe : certains blasons font l'objet d'une transmission à l'intérieur de la société - d'humains à humains -, tandis que d'autres, les plus prestigieux, doivent leur origine à une transmission à un ancêtre (mythique ou mytho-historique) par un être surnaturel qui s'est manifesté sous une apparence animale. C'est seulement à

34 De Laguna (172: 458) indique que des fusils russes pris par un clan tlingit se virent attribués des noms blasonnés : Fusil Ours, Fusil Epaulard. Un guerrier peut accaparer pour lui-même le motif blasonné peint sur le visage de l'homme qu'il a tué au combat. Quand il est pris de force, le blason ne peut être montré en public, à moins que son ancien propriétaire ne l'ait racheté. 
l'occasion de ces cérémonies qu'un objet héraldique acquiert toute son importance et toute sa valeur.

Le système à blasons peut être envisagé comme un moyen d'identification des groupes et de repérage de la position de chacun d'eux par rapport à un ensemble plus ou moins large de groupes partenaires de même niveau hiérarchique ou de niveau inférieur. On pourrait parler tout autant d'un dispositif de régulation des relations entre les groupes. Cette conception n'est pas éloignée de celle de Boelscher (1988 : 150), qui écrit à propos des Haida :

" Les blasons véhiculent des messages relatifs à l'ordonnancement des relations sociales. Cependant ces messages ne mettent pas l'accent sur le blason en tant que symbole de différenciation dans un sens purement classificatoire, mais ils le constituent comme symbole des transactions [entre les groupes]. Ils [les blasons] reflètent l'histoire des relations entre les personnes et les lignages dans la mesure où ils symbolisent la réussite des détenteurs de blasons dans l'accumulation d'un capital matériel et symbolique ".

Halpin (1973, 1984), qui inscrit ses propos dans le sillage de LéviStrauss, fait discrètement retour au paradigme totémique. Cet auteur considère que les blasons animaux relèvent soit d'une logique de différenciation, soit d'une logique de hiérarchisation, ce qu'elle illustre par un système de coordonnées à deux axes, l'axe horizontal étant associé à la première de ces deux logiques, l'axe vertical à la seconde. L'opposition entre les deux axes renvoie à celle déjà rencontrée entre blason générique et blason particularisé. En vérité, comme le montre bien M. Halpin, cette opposition n'est pertinente que pour les Tsimshian ; chez les Haida et les Tlingit, où le système hiérarchique est peu évolué, il y a prévalence des faits de différenciation sur ceux de hiérarchisation. En dépit de sa portée limitée, l'analyse de Halpin met en lumière une équivalence formelle entre les blasons malgré leurs différences, lesquelles trouvent leur origine dans l'histoire et peuvent renvoyer à toute une série de déterminations particulières : situation hiérarchique et donc prestige acquis, plus ou moins grande ancienneté, plus ou moins grande imprégnation de sacralité, etc. C'est peut-être cette concurrence entre la structure et l'histoire - nous en avons évoqué des manifestations récentes - qui met le plus à mal le bel ordonnancement totémique. 
Pour bien comprendre la nature de notre interrogation finale sur le blason, il convient de prendre au sérieux ce fait d'évidence : si les dénominations des blasons sont principalement animales, elles ne le sont pas en totalité et l'éventail d'ensemble des dénominations, considérées indépendamment de leur fréquence, concerne, comme on l'a vu, une grande variété d'êtres ou d'objets. Dès lors, on chercherait en vain à identifier la nature profonde du blason, sauf à en faire un élément de ce que M. Boelscher appelle un " capital symbolique et matériel ". C'est là un point crucial : le blason est un bien, à la fois réel et symbolique, matériel et spirituel, dont on a vu qu'il se situe à l'articulation du mythe et de l'histoire, qu'il établit une relation permanente entre le monde social et son environnement naturel et surnaturel, qu'il a à voir aussi bien avec la territorialité et la formation des communautés locales qu'avec l'idéologie du prestige, qu'en général, ou sous certaines conditions, il circule, est transmissible, aliénable, captable, ces différentes caractéristiques étant assujetties au fait qu'il est en permanence approprié et que cette appropriation est continuellement en instance de validation. Ainsi l'analyse du système à blasons nous fait-elle retrouver le génie propre des cultures de la côte Nord-Ouest.

Marie Mauzé C.N.R.S. 


\section{Références bibliographiques}

Adam, Leonard

1913 "Stammesorganisation und Häuptlingstum der Haida und Tsimshian ", Zeitschrift für vergleichende Rechtswissenschaft 30: 161-268.

Barbeau, Marius

1917 "Growth and Federation in the Tsimshian Phratries ", Proceedings of the Nineteenth International Congress of Americanists, 1915. Washington, pp. 403-408.

1929 Totem Poles of the Gitskan, Upper Skeena River, British Columbia. Ottawa, National Museum of Canada, Bulletin $n^{\circ}$ 61.

1939 "How Totem Poles Originated ", Queens Quaterly 46 (3) : 304 311.

1940 "The Modern Growth of the Totem Pole on the Northwest Coast ", Smithsonian Institution Annual Report 1939: 491-498.

1942 "Totem Poles : A By-product of the Fur Trade ", Scientific Monthly, December : 504-514.

1944 "Totemism. A Modern Growth on the North Pacific Coast ", Journal of the American Folklore $57: 223$.

Boas, Franz

1889 "First General Report on the Indians of British Columbia ", Report of the British Association for the Advancement of Science, pp. 801-893.

1890 "The Use of Masks and Head Ornaments on the Northwest Coast of America" Internationales Archiv für Ethnographie : 715.

1897a "The Decorative Art of the Indians of the North Pacific Coast ", Bulletin of the American Museum of Natural History 9 : 123-176.

1897b The Social Organization and Secret Societies of the Kwakiutl Indians. United States National Museum. Report for 1895 : 311 . 738.

1898 "Twelfth And Final Report on the Norhwestern Tribes of Canada ", British Association for the Advancement of Science : 40-55.

1910a [1974] " Psychological Problems in Anthropology ", American Journal of Psychology 21 : 371-384, in George Stocking, ed., 1974, A Franz Boas Reader. The Shaping of American Anthropology 1883-1911. Chicago, University of Chicago Press, pp. 243-254. 
$1910 \mathrm{~b}$ "The Origin of Totemism ", Journal of the American Folklore XXIII : 392-393.

1916a "The Origin of Totemism ", American Anthropologist 18 : 319 326

$1916 \mathrm{~b}$ "Tsimshian Mythology". 31st Annual Report of the Bureau of American Ethnology for 1909-1910. Washington D.C. Governement Printing Office.

1920 "The Social Organization of the Kwakiutl Indians ", American Anthropologist, n.s. 2:111-126.

1966 Kwakiutl Ethnography. Edited by Helen Codere. Chicago. University of Chicago Press.

Boelscher, Marianne

1988 The Curtain Within. Haida Social and Mythical Discourse. Vancouver, University of British Columbia.

Chapman, Anne

1965 Mâts totémiques. Paris, Muséum d'histoire naturelle. Catalogue du Musée de l'Homme.

Chowning, Ann

1962 "Raven Myths in the North-Western North America and North-Eastern Asia ", Arctic Anthropology 1 (1) : 1 -5.

Cole, Douglas et Bradley Lockner (éds.)

1993 To the Charlottes. George Dawson's 1878 Survey of the Queen Charlotte Islands. Vancouver, UBC Press.

Cove, John

1987 Shattered Images. Dialogues and Meditations on Tsimshian Narratives. Ottawa, Carleton University Press.

Dauenhauer, Nora Marks et Richard Dauenhauer, (éds.)

1994 Haa Kusteeyi, Our Culture. Tlingit Life Stories. Seattle, University of Washington Press.

Davy, Georges

1922 La Foi jurée : étude sociologique du problème de contrat, la Descola, Philippe formation dulien contractuel. Paris, Librairie Félix Alcan.

1996 "Constructing Natures. Symbolic Ecology and Social Practice " in P. Descola et Gisli Pàlsson (éds.), Nature and Society. London, Routledge, pp. 82-102.

Downie, R.A.

1970 Frazer and the Golden Bough. London, Gollancz.

Duff, Wilson

1964 " Contributions of Marius Barbeau to West Coast Ethnology ", Anthropologica 6 (1) : 63-96. 
Dunn, John

1984 "International Matri-moities : The North Maritime Province of the North Pacific Coast " in Margaret Seguin (éds.), The Tsimshian. Images of the Past; Views for the Present. Vancouver, University of British Columbia, pp. 99-109.

Durkheim, Emile

1900 " Analyse des travaux (...): 'The social Organization and the Secret Societies of the Kwakiutl Indians' ", L'année sociologique 1898-1899 : 336-340.

1912 Les formes élémentaires de la vie religieuse. Paris, Librairie Félix Alcan.

Duthuit, Georges

1974 [1946] * Le don indien sur la côte Nord-Ouest de l'Amérique (Colombie britannique) " in Représentation et présence. Premiers écrits et travaux 192.3-1952. Paris, Flammarion, coll. "Idées et recherches " pp. 315-319.

Emmons, George Thornton

1991 The Tlingit Indians. Edited with additions by Frederica de Laguna. Seattle, University of Washington Press; New York, American Museum of Natural History.

Frazer, J. G.

1887 Totemism. Edinburgh, Adam \& Charles Black.

1922 Les origines de la famille et du clan. Paris, Geuthner. Ed. Orig., Totemism and Exogamy, 4 vols. London, 1910.

Garfield, Viola

1939 Tsimshian Clan and Society. Seattle, University of Washington Press, University of Washington Press Publications in Anthropology, vol $7 n^{\circ} 3$.

1951 The Tsimshian and their Arts. The Tsimshian and their Neighbors. Seattle, University of Washington Press.

Goldenweiser, Alexander

1910 "Totemism : An Analytical Study ", Journal of American Folklore XXIII : 179-292.

1912a "Andrew Lang on Method in the Study of Totemism ", American Anthropologist 14 : 382-391.

1912b "The Origin of Totemism ", American Antbropologist $14: 600$ 607.

1918 "Form and Content in Totemism ", American Anthropologist $20: 280-295$.

Guédon, Marie-Françoise

1984 "An Introduction to Tsimshian Worldview and its Practitioners ", in Margaret Seguin, ed. The Tsimshian. Images 
of the Past; Views for the Present. Vancouver, University of Haekel, Joseph

British Columbia, pp. 137-159.

1983 "Totemism " in The New Encyclopedia Britannica, pp. 529-533.

Halpin, Marjorie

1983 Totem Poles. An Illustrated Guide. Vancouver, University of British Columbia Press in association with the U.B.C Museum.

1973 The Tsimshian Crest System : A Study Based on Museum Specimens and the Marius Barbeau and William Beynon FieldNotes. PHD. Dissertation, Vancouver, University of British Columbia.

1984 "The Structure of Tsimshian Totemism ", in Jay Miller and Carol M. Eastman (éds.), The Tsimshian and their Neigbbors. Seattle, University of Washington Press, pp. 17-35.

Hill-Tout, Charles

" The Origin of Totemism of the Aborigines of British Columbia " in Proceedings and Transactions of the Royal Society

Jonaitis, Aldona of Canada, 2nd series, VII.

1986 Art of the Northern Tlingit. Seattle, University of Washington Press.

1989 "Mâts totémiques et New Deal ", Gradhiva 6 : 13-22.

Jorion, Paul

1985 "Totemism " in Adam Kuper and Jessica Kuper, eds., The Social Science Encyclopedia. London, Routledge, pp. 860-861.

Kan, Sergei

1989 Symbolic Immortality. The Tlingit Potlatch of the Nineteenth Century. Washington, Smithsonian Institution Press.

Kuper, Adam

1988 The Invention of Primitive Society. Transformations of an Illusion. London, Routledge.

Laguna (de) Frederica

1952 "Some Dynamics Forces in Tlingit Society ", Southwestern Journal of Anthropology 10 (2) : 1-12.

1975 "Matrilineal Kin Groups in Northwestern North America ", in A. McFayden Clark (éds.), Proceedings : Northern Athapaskan Conference, 1971, Ottawa, National Museums of Canada, vol 1., pp. 17-145.

1972 Under Mount Saint Elias : The History and Culture of the Yakutat Tingit. Smithsonian Contributions to Anthropology 7, Parts 1, 2, 3, Washington. 
Lévi-Strauss, Claude

1962a La Pensée sauvage. Paris, Plon.

1962b Le Totémisme aujourd'hui. Paris, Puf.

1979 La Voie des masques, edition revue, augmentée et rallongée de trois excursions. Paris, Plon.

1989 Des Symboles et leurs doubles. Paris, Plon.

Lowie, Robert

1960 [1911] "A New Conception of Totemism " in Cora Du Bois (éds.),

Lowie's Selected Papers in Anthropology. Berkeley, University of

California Press, pp 293-311.

MacDonald George

1983 Haida Monumental Art. Vancouver, University of British Columbia Press.

Mauss, Marcel

1947 Manuel d'ethnographie. Paris, Payot.

1969 Oeuvres. 3. Cohésion sociale et division de la sociologie. Paris, Editions de Minuit.

McC. Grubb, David

1977 A Practical Writing System and Short Dictionary of Kwakw'ala (Kwakiutl). Ottawa, National Museum of Man, Mercury Series.

McLennan, J. M.

1869 "The Worship of Animals and Plants ", The Fortnightly Review 6 : 407-582 ; $7: 194216$.

Miller, Jay

1997 Tsimshian Culture. A Light through the Ages. Lincoln, University of Nebraska Press.

Miller Jay \& Carol M. Eastman, (éds.)

1884 The Tsimshian and their Neighbors, Seattle, University of Washington Press

Moret A. et G. Davy

1923 Des Clans aux empires. Paris, La Renaissance du livre.

Murdock, George P.

1936 Rank and Potlatch among the Haida. Yale University Publications in Anthropology, 13. New Haven, Yale University Press.

Niblack, Albert

1970 [1890] The Coast Indians of Southern Alaska and North British

Oberg, Kalervo

$$
\text { Columbia. New York, Johnson Reprint. }
$$

1973 The Social Economy of the Tlingit Indians. Seattle, University of Washington Press; Vancouver, Douglas \& McIntyre. 
Radcliffe-Brown, A. R.

1968 Structure et fonction dans la société primitive. Paris, Les Editions de Minuit.

Reichards, Gladys

1938 [1965], "Totemism" in Franz Boas (éd.), General Anthropology. Boston, D.C. Heath and Company, pp. 426-430.

Sapir Edward

1915 [1965] "Social Organization of the West Coast Tribes ", in A. L. Kroeber and T.T. Waterman (éds.), Source Book in Anthropology. New York, Johnson Reprint Corporation, pP 317-333..

Seguin, Margaret (éd.)

1984 "Introduction: Tsimshian Society and Culture " in M. Seguin (éd.), The Tsimshian. Images of the Past; views for the Present. Vancouver, University of British Columbia, pp. ix-xx.

Shapiro, Warren

1991 Claude Lévi-Strauss Meets Alexander Goldenweiser : Boasian Anthropology and the Study of Totemism ", American Anthropologist 93 : 599-610.

Shore, Bradd

1989 "Totem as Practically Reason : Food for Thought ", Dialectical Anthropology 14 (3) : 177-195.

Stewart, Hilary

1993 Looking at Totem Poles. Préface de Norman Tait. Vancouver, Douglas \& McIntyre ; Seattle, University of Washington Press.

Swanton, John R.

1904 "The Development of the Clan System and Secret Societies among the Northwestern Tribes ", American Anthropologist, n.s., $6: 477-485$.

1905a Contributions to the Ethnology of the Haida. American Museum of Natural History Memoir 8 (1).

1905b "The Social Organization of American Tribes ", American Anthropologist $7:$ 663-673.

1908 "Social Conditions, Beliefs, and Linguistic Relationship of the Tlingit Indians ", Twenty-Sixth Annual Report of the Bureau of American Ethnology for the Years 1904-1905: 391-512. Washington, US Governement Printing Office.

1910 "Totem "; " Totem Poles ", in Frederick Webb Hodge (éd.), Handbook of the American Indians North of Mexico. Smithsonian Institution, Bureau of American Ethnology Bulletin 30. Washington, Government Printing office, pp. 787795. 
Testart, Alain

1981 "Totem ", Enciclopedia, tome XIV, Torino, Einaudi, pp. 388413.

1985 Le communisme primitif. Economie et idéologie. Paris, Editions de la Maison des sciences de l'homme.

Van Gennep, A.

1920 L'Etat actuel du totémisme. Paris. Editions Ernest Leroux.

Voget, W. Fred

1975 A History of Ethnology. New York, Holt, Rinehart and Winston.

Wagner, Roy

1987 "Totemism " in Encyclopedia of Religion 14 : 573-576.

Winthrop, Robert $\mathrm{H}$.

1991 "Totemism " in Dictionary of Concepts in Cultural Anthropology, New York, Greenwood Press, pp. 297-299. 